\title{
Seismic hazard due to fluid injections
}

\author{
Cole Lord-May, ${ }^{1}$ Jordi Baró $\odot,{ }^{1,2,3}$ David W. Eaton, ${ }^{2}$ and Jörn Davidsen ${ }^{1,4, *}$ \\ ${ }^{1}$ Complexity Science Group, Department of Physics and Astronomy, University of Calgary, 2500 University Drive NW, \\ Calgary, Alberta, Canada T2N $1 N 4$ \\ ${ }^{2}$ Department of Geosciences, University of Calgary, 2500 University Drive NW, Calgary, Alberta, Canada T2N 1N4 \\ ${ }^{3}$ Centre for Mathematical Research, Bellaterra, Barcelona 08193, Spain \\ ${ }^{4}$ Hotchkiss Brain Institute, University of Calgary, 3330 Hospital Drive NW, Calgary, Alberta, Canada T2N 4N1
}

(Received 27 November 2019; revised 26 May 2020; accepted 23 November 2020; published 7 December 2020)

\begin{abstract}
Earthquakes can be induced by natural and anthropogenic processes involving the injection or migration of fluids within rock formations. A variety of field observations has led to the formulation of three different and apparently contradicting paradigms in the estimation of the seismic hazard associated with fluid injections. We introduce a unified conceptual model accounting for the nonhomogeneous pore-pressure stimulation caused by fluid injection in a prestressed region, to prove how all three paradigms can naturally coexist. Within our model framework the loading history, accounting for both the fluid injections and natural tectonic loading, together with the heterogeneity of the host medium determine which of the three paradigms prevails at a given time. We identify a superposition of two populations of events triggered at different local stress levels with different Gutenberg-Richter $b$ values. Our findings suggest that both the observed diversity of $b$ values across different fluid-induced settings and the dominating hazard paradigm are a consequence of such a superposition leading to an effective $b$ value.
\end{abstract}

DOI: 10.1103/PhysRevResearch.2.043324

\section{INTRODUCTION}

Variations in pore pressure due to fluid invasion in stressed materials can activate processes of mechanical failure, such as the initiation, propagation, and coalescence of fractures or the slip of frictional surfaces in prefractured faults [1-3]. This phenomenon accounts for (micro-)seismic activity recorded in natural and stimulated geothermal systems [enhanced geothermal system (EGS)] [4-8], during hydraulic fracturing (HF) operations [9-11], and following the underground disposal of wastewater [12]. Conceptual models explain how failure can be induced by variations in the applied stress tensor and pore pressure stimulated by highly pressurized fluid injection [13-16]. The typical magnitudes of fluid-induced seismicity are low (magnitudes $m \leqslant 0$ ), which is often challenging to detect on the surface and well below the threshold for being felt [17]. However, in some rare cases these processes can activate nearby faults and trigger stronger events [18-20]. One of the most remarkable and intriguing features of fluid-induced seismicity is a high diversity in the $b$ value defining the Gutenberg-Richter (GR) distribution:

$$
\operatorname{pdf}(m) d m=\frac{1}{b \ln 10} 10^{-b\left(m-m_{c}\right)} d m,
$$

\footnotetext{
*davidsen@phas.ucalgary.ca

Published by the American Physical Society under the terms of the Creative Commons Attribution 4.0 International license. Further distribution of this work must maintain attribution to the author(s) and the published article's title, journal citation, and DOI.
}

for seismic events with magnitudes $m$ above the magnitude of completeness $m_{c}$ of a given seismic catalog (where pdf is the probability density function). While tectonic processes render $b \sim 1$ with some variability across different settings [21-25], the distribution of $m$ in fluid-induced seismicity is compatible with a wide spectrum of $b$ values ranging from significantly less than 1 to much larger than 2 for different areas and/or time periods $[26,27]$. This together with other field observations such as the spatiotemporal evolution of seismic activity around the stimulation front, a lack of large-magnitude events, and dependencies of event sizes on the cumulative volume of injected fluid over time $V(t)$ has led to three different and apparently contradictory paradigms in the estimation of the seismic hazard associated with fluid injections [28], as summarized in Fig. 1:

(a) Paradigm A. Based on observations [26,29] and energy balance assumptions [30,31], A-type hazard models link fluidinduced seismicity to the activation of small fractures inside the stimulated reservoir. Microseismic events are contained within the stimulated area and occur upon the activation of inactive preexisting fractures (see top panel of Fig. 1), which partially determine a time-independent distribution of magnitudes or seismic moments [26,32]. The number of susceptible fractures and the probability of activation are proportional to the injected volume $[26,30,32,33]$ or implicitly constrained by the energy supplied [31] and locally increase with pore pressure [26]. All A-type hazard models commonly assume a stationary GR distribution. Hence the number of fractures above a magnitude $m_{0}$ as a function of injected volume over time $V(t)$ reads

$$
N_{>m_{0}}(t)=10^{\Sigma} V(t) P\left(m>m_{0}\right),
$$




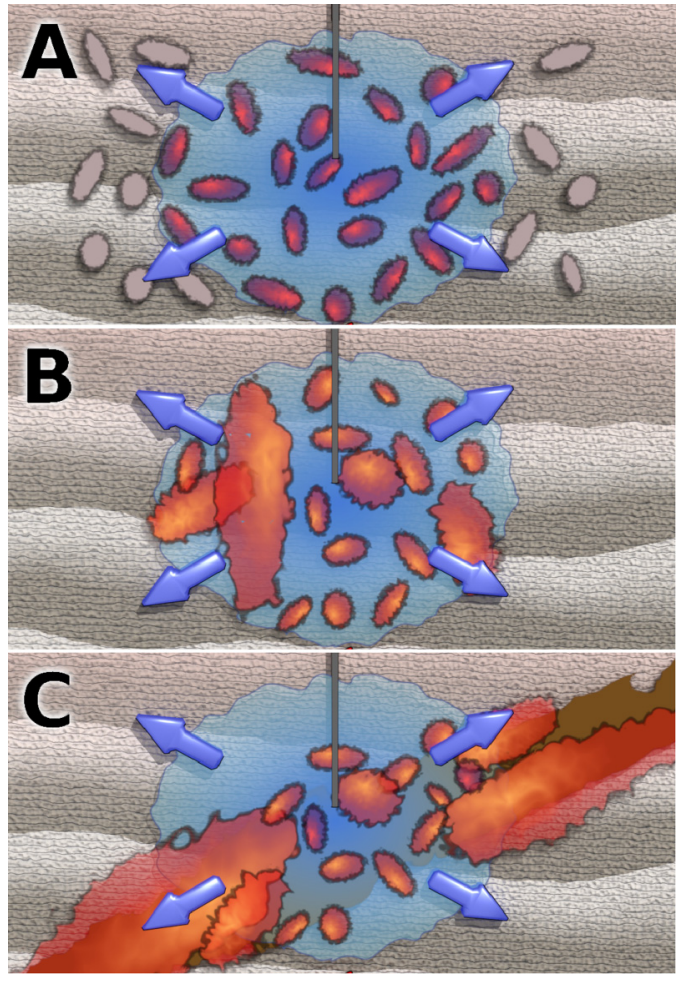

FIG. 1. Schematic representation of the physics behind the three hazard-model paradigms (paradigms A-C). Blue areas represent the expanding stimulated area. Gray patches are inactive preexisting fractures, while red patches represent wet fractures activated by changes in pore pressure.

where the cumulative magnitude distribution $P\left(m>m_{0}\right)=$ $10^{-b\left(m_{0}-m_{c}\right)}$ is stationary. The seismogenic index $\Sigma$ completely determines the hazard rates and is observed to be constant over time in several HF and EGS operations [32]. The maximum magnitude in a sequence of seismic events is determined by record statistics, and its expected value can be estimated by inverting $N_{>m_{0}}(t)=1$. Other models within the same paradigm impose constraints on the supplied energy, giving rise to time dependencies (but not magnitude dependencies) of $\Sigma$ [31].

(b) Paradigm B. Other field studies [34,35] have revealed a dependence between the possible event magnitudes and injected volumes. B-type hazard models interpret this observation as follows. Dynamic or preexisting fractures have a typical GR distribution with low $b$ values; that is, large events are statistically relevant. However, fractures are constrained in size to fit within the stimulated area (see middle panel of Fig. 1); that is, there exists a maximum fracture area that increases over time in proportion to the total volume injected, resulting in a GR distribution with a cutoff for large $m$ [28]. The effective seismogenic index $\Sigma$ is magnitude dependent in this case and evolves over time in a nonscaling relation with $m_{0}$. In particular, considering the $b$ value fitted from the preceding activity, $\Sigma$ underestimates the hazard of large earthquakes at long times and, having otherwise access to the $b$ value at later times or considering just small events, overestimates the hazard at early times [34]. This is caused by the existence of a characteristic size $m^{*}(V) \sim \eta \log V$ with a scaling exponent $\eta$ that determines the seismic hazard and the maximum magnitude instead of the $b$ value. Large fluidinduced seismic events in HF and EGS operations are often better described within this paradigm [34].

(c) Paradigm $C$. Some seismic activity is triggered, rather than induced, by fluid injections, meaning that natural (tectonic) activity is simply accelerated [36]. C-type hazard models consider those events to be indistinguishable from tectonic earthquakes, which means that they can expand without any constraint imposed by the size of the stimulated area (see bottom panel of Fig. 1). The hazard assessment is based on the (tectonic) GR distribution (1) determined by the tectonic setting [37]. The injected volume only controls the total number of earthquakes rather than the total seismic moment, following the same arguments provided in Ref. [26]. The expected maximum magnitude is again determined by record statistics of a stationary distribution but with $b$ values typical for tectonic events, which are usually lower than for induced seismicity.

While all three modeling paradigms have proven useful to assess the seismic hazard in specific case studies, their predictions are diametrically opposed. In particular, it has remained unclear why one paradigm should prevail over another one in a given situation. Here, we tackle this question from a conceptual microscopic model perspective. Specifically, to establish how these hazard paradigms can arise from the underlying dynamics, we focus on the potential role of interactions involving fluid dynamics and fractures and introduce a minimal dynamical model of stick-slip micromechanics as the source of the fractures, which is driven by the injected fluid. We find that our conceptual model can reproduce the main observed features of fluid-induced seismicity with respect to the associated seismic hazard (paradigms A-C), including the diversity in the $b$ value. In particular, it contrasts the effect of two different event populations - one arising in (locally) predominantly low-stress situations or in a highly heterogeneous medium and the other one arising in (locally) predominantly high-stress situations or in a more homogeneous medium-and sheds light on their interplay.

\section{MODEL}

Geomechanical fracture processes can be simplified as a one-dimensional Mohr-Coulomb problem [38]. Each representative volume element (RVE) of the material at position $r$ is mechanically unstable when the minimum principal stress $\tau_{s, r}$ surpasses a stability limit:

$$
\tau_{s, r} \geqslant \tau_{c, r}+\mu_{r}\left(p_{0, r}-p_{p, r}\right),
$$

where $\tau_{c, r}$ is the minimum cohesive force needed to fracture the material, $\mu_{r}$ is the static friction coefficient, $p_{0, r}$ is the local normal stress, equal to the confining pressure under the assumed conditions over the RVE, and $p_{p, r}$ is the internal pore pressure due to the presence of fluids. Given a fixed hydrostatic pressure and cohesive force, failure can occur by either an increase in the anisotropy of the applied forces and, hence, in $\tau_{s, r}$ (also known as dry fracture [18]) or an increase in the pore pressure $p_{p, r}$ caused by internal fluid dynamics (wet fracture) [39] such as the injection of a fluid $\alpha$ in a region saturated with a fluid $\beta$. A fracture initiated at $r$ can propagate and grow through internal interactions. The local 
stress $\Delta \tau_{s, r}$ is released and (partially) distributed to other RVEs at $r^{\prime}: \tau_{s, r^{\prime}} \rightarrow \tau_{s, r^{\prime}}+a\left(r-r^{\prime}\right) \Delta \tau_{s, r}$, where $a\left(r-r^{\prime}\right)$ is a factor depending on the interactions and dissipation.

Our model represents the RVE as point sites $(i, j, \ldots)$ in a two-dimensional (2D) square lattice. The evolution of the $p_{p}$ profile is implemented as a generalized fluid model with a tunable pressure-gradient profile, which allows us to incorporate both point diffusion and invasion percolation-two scenarios typically considered $[9,40]$ - as end members such that our model provides a unified description of fluid invasion.

\section{A. Pore-pressure stimulation model}

Each RVE contains either a reservoir fluid $\beta\left(p_{p, \beta}=0\right)$ or invasion fluid $\alpha$ with concentrations 0 or 1 . A local resistance to flow $v_{i, j}$, accounting for capillarity and poromechanics at the invading front, links each neighboring site $i, j$. In this case, we choose $v_{i, j}$ to be stationary in time, uncorrelated and uniformly distributed between 0 and 1 . Assuming that the fluid $\alpha$ is incompressible and the injection rate at the point source is constant, at each time step the fluid $\alpha$ displaces the fluid $\beta$ in a site $j$ by invasion from a neighboring site $i$ and increases the volume $V$ of fluid $\alpha$ by one unit. The invaded site is selected from the highest value $p_{p, i}-p_{p, j}+v_{i, j}$ of all $\{i, j\}$ pairs in the fluid interface. The shape of the invading front is controlled by the constant pressure $p_{p \text {, min }}>0$ at the longest path length $l_{M}$ from the injection site to the interface. The pressure profile in $\alpha$ is modeled as an exponential decay from 1 to $p_{p, \min }$ as a function of path length $l$ from the injection site:

$$
p_{p, \alpha}(l)=p_{p, \min }^{l / l_{M}}
$$

The parameter $p_{p, \min }$ not only determines the minimum pore pressure at invaded sites but also is one of the key parameters in our conceptual model. In particular, it allows us to mimic the dynamics of both point diffusion and invasion percolation as end members: $p_{p, \text { min }} \approx 0$ corresponds to the case when viscous forces dominate over capillary forces giving rise to a point-diffusion process $[9,41] . p_{p, \min }=1$ corresponds to the opposite case giving rise to invasion percolation [40]. Indeed, the viscosity of the internal fluids of the reservoir and the poromechanics of the specific geological setting can give rise to these two conceptually opposed behaviors of fluid invasion:

(1) When viscous forces dominate and the medium is poroelastic, the stimulated reservoir can be modeled by Darcy's law within a homogeneous medium [42], rendering a point-diffusion process where the propagation of the invading fluid depends on the metric distance from injection. Darcy's law describes empirically the fluid propagation within a porous medium. According to Darcy's law, the average flux of the invading fluid, $\vec{\phi}_{\alpha}$, is then given by

$$
\vec{\phi}_{\alpha}=-\frac{k_{r, \alpha}}{\eta_{\alpha}} \vec{\nabla} p_{p, \alpha},
$$

where $k_{r, \alpha}$ is the effective permeability for the invading fluid $\alpha$ in the porous medium, $\eta_{\alpha}$ is its viscosity, and $\vec{\nabla} p_{p, \alpha}$ is the pressure gradient. The actual pore-pressure profile depends on geomechanical factors such as the poroelasticity of the medium. The selected exponential pore-pressure profile is a conceptual simplification. Our findings are not specific to this choice and are robust against more complex, yet still smooth, profiles expected in poroelastic reservoirs [9]. A linearly decaying profile as well as an inverse profile as a function of distance were tested as extreme cases and led to indistinguishable results.

(2) When capillarity dominates or when brittle poromechanics are considered-meaning that a pressure gradient is needed to unclog the pores and access new regions of the reservoir in a brittle process [40] - the effect of viscous forces is negligible, and the stimulated reservoir is better modeled by invasion-percolation models [43], where the fluid explores a lattice of interconnected channels representing the host porous medium. In this case, the propagation of the invading fluid does not depend on the distance to the source and is entirely determined by the heterogeneity of the host porous medium, rendering fractal structures.

Both conceptual approaches might be valid in some specific situations; yet our proposed unified model captures both situations as end members, thus allowing a more general description.

\section{B. Stick-slip microseismic model}

Microseismic events are simulated through a spring-block model considering only shear stick-slip processes; that is, tensile opening mechanisms are omitted [44]. The failure condition in Eq. (3) is simplified as stick-slip dynamics

$$
\tau_{s, i} \geqslant s_{i}-p_{p, i},
$$

where $\tau_{s, i}$ is the local principal stress at site $i$ normalized by the friction and $s_{i}:=\tau_{c, r} / \mu_{r}+p_{0, r}$ accounts for the joint contribution of the cohesive force, the confinement pressure, and friction. On failure, all the local stress is released $\left(\Delta \tau_{s, i}=\tau_{s, i}\right)$ and distributed equally to the nearest neighbors. Since $\max \left(p_{p}\right)=1, s_{i}$ values must be bounded to be above a value $s_{\min }>1$ to guarantee stable jamming in the stick-slip dynamics. In this case, we choose the $s_{i}$ values to be stationary in time, spatially uncorrelated, and uniformly distributed between $s_{\min }$ and $s_{\max }$ determining the stress level and the heterogeneity of the frictional medium. In general terms, a tectonically active zone will be closer to failure than an inactive one, and the stress levels due to external driving $\left(\tau_{s, i}\right)$ will be, on average, closer to $s_{i}$ (low $s_{\min }$ and $s_{\max }$ ). In contrast, high $s_{\max }$ values will denote low stress levels or regions with low residual stress from none to low natural tectonic activity. Thus $s_{\min }$ and $s_{\max }$ determine the range of the strength field and are the other key parameters in our model in addition to $p_{p, \min }$.

\section{Simulation details}

The results presented below are for the case of a constant quasistatic injection of fluid $\alpha$ at a point inside an open reservoir, originally saturated with fluid $\beta$, as typically encountered in not only stimulated geothermal systems but also similar HF operations. Local shear stresses $\tau_{s, i}$ only change by slip or site interactions, neglecting slow tectonic loading. Fractures initiate at the onset of an instability [see Eq. (6)] caused exclusively by wet mechanisms ( $p_{p}$ evolution) and propagate by transfer of $\tau_{s, i}$, while the $p_{p}$ profile is kept stationary. 
In summary, our model has four associated fields: the resistance-to-flow field $v$, the pore-pressure field $p_{p}$, the stress field $\tau_{s}$, and a strength field $s$. The resistance-to-flow field and the strength field are stationary over time and take random values as explained below. The other two fields change dynamically with the following simulation procedure:

(i) Simulations are initialized by assigning resistance-toflow values from the uniform distribution $v_{i, j} \in[0,1)$ to bonds adjacent to the injection site which define the fluid interface. We assign $s_{i} \in\left[s_{\min }, s_{\max }\right)$ and initial $\tau_{s, i} \in[0,1)$ to the source. We allow the simulation to run without size restriction-corresponding to an open reservoir with no boundaries or size limit - and the three uncorrelated fields are generated on the fly to speed up the simulation.

(ii) At each injection time step, fluid flows through the highest $p_{p, i}-p_{p, j}+v_{i, j}$ of all $i, j$ pairs along the fluid interface, following the nonhomogeneous percolation problem described above with nontrapping rules [45]. If this increases the maximal path length $l_{M}$, we update the pore-pressure field, increasing the pressure at all stimulated sites. The $p_{p, j}$ values are assigned according to the path length from the origin $l_{j}$ following relation (4). Nonstimulated sites remain at an arbitrary pressure value $p_{p, j}=0$.

(iii) At each injection time step, we check for sites satisfying the failure condition (6). If the newly invaded site does not increase $l_{M}$, only that site needs to be checked. Otherwise, all invaded sites need to be checked. Upon failure, we distribute a fixed fraction of $\tau_{s, i}$ equally among the four neighboring sites $j$ and set $\tau_{s, i}=0$, such that the conservative case corresponds to the fixed fraction being 1 . Within the same injection step, all neighboring sites $j$ are checked for failure, and stress is redistributed. We simultaneously check the failure condition for all neighbors of those failed neighbors before redistributing the stress again. This process is repeated until no more sites satisfy the failure condition. The spatial location and characteristics are recorded. In the instance where multiple sites satisfy the initial failure condition due to an increase in pressure, the site with the greatest $\tau_{s, i}+p_{p, i}-s_{i}$ slips and propagates the stress, and the failure condition is rechecked for the rest of the candidates afterwards.

(iv) Once no more slips occur and no more stress needs to be redistributed, we resume injection and return to step (ii).

Simulations are performed on a 2D square lattice with nearest-neighbor interactions for both fracture and fluid propagation. The size of the lattice is dynamically allocated with the propagation of the fluid and fractures. The simulation results presented here reach sizes of around 30000000 stimulated sites and lattices up to $50000000(\approx 7000 \times 7000)$ sites such that a maximum of $N=10^{6}$ fracture events are recorded. To conclude, the three relevant parameters are as follows: $p_{p, \min }$ determines the minimum pore pressure at invaded sites, while $s_{\min }$ and $s_{\max }$ determine the range of the strength field. We select $s_{\min }=1.3$ and $p_{p \text {, min }}=0.3$ while varying $s_{\max }$ between 1.4 and 2.3. Yet other parameter values have been studied as well showing that our findings are robust.

\section{RESULTS}

We distinguish three metrics to define the size of the fracture. The fracture area $A$ is measured as the number of unique

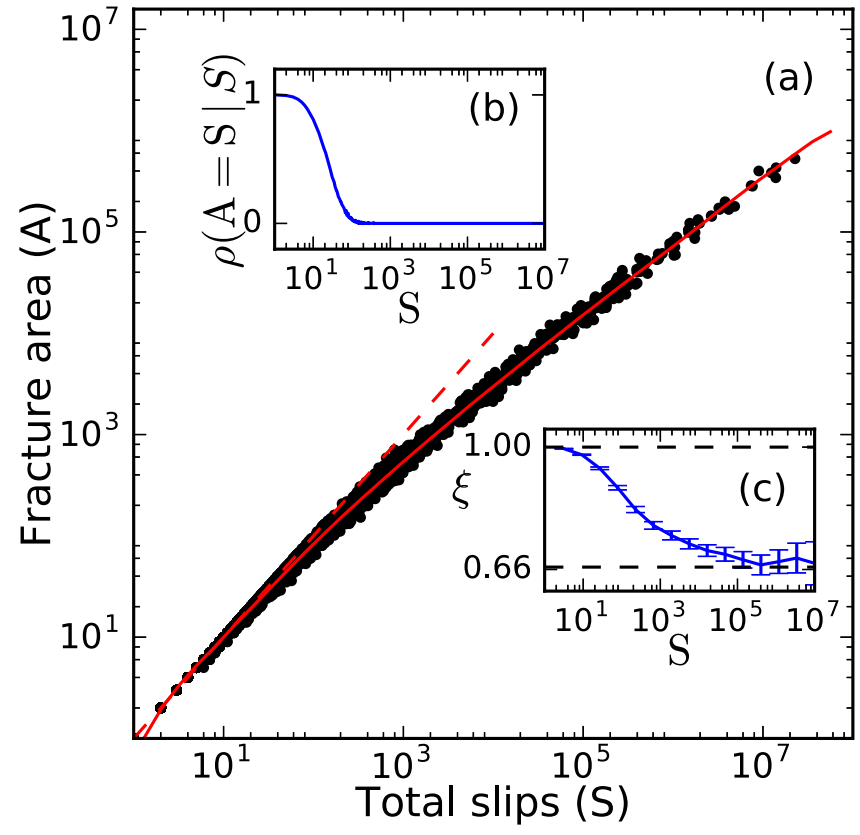

FIG. 2. (a) Scatterplot of fracture area $A$ against total slips $S$ for $p_{\min }=0.3, s_{\min }=1.3$, and $s_{\max }=1.4$. The solid red curve shows the moving average $\langle A\rangle(S)$. (b) Density $\rho$ of single-slip events $(S=A)$ matching the dashed line in (a), as a function of $S$. (c) Exponent $\xi(S)$ estimated from a linear fit in moving intervals.

sites that slip within the same fracture. The seismic moment $M_{0}$ of the events is usually assumed to be proportional to the total number of individual slips $S$. Potency $P_{0}$ is claimed to effectively relate field data with models [46] and is assumed to be proportional to the total stress released $\left(\Delta=\sum_{i \in \text { event }} \tau_{s, i}\right)$. On average, $S \propto \Delta$ in our model, consistent with a constant shear modulus $\mu$ and indicating that the micromechanics are similar for all events. Similarly, fracture area and moment are expected to be related on average such that $\langle A\rangle(S) \sim S^{\xi}$ [47]. Models with short-range interactions exhibit a transition between $\xi=2 / 3$ and $\xi=1$ for small events, imposing a characteristic scale on the size relation. Figure 2(a) illustrates this effect in our model in a scatterplot of $A$ versus $S$. The exponent transition is smooth as in related models [39] extending significantly beyond the range where events with $S=$ $A$ are observed [see Fig. 2(b)]. Previous studies related such a smooth transition to evolving heterogeneities [48], which are introduced in our model by the pore-pressure profile. We find that the scaling relations between $A, S$, and $\Delta$ are invariant with respect to variations in the simulation parameters within the ranges explored in this paper and with respect to relevant subpopulations of events (not shown).

Despite the characteristic scale in the relation $A(S)$, the event size distributions for all three metrics are compatible with power laws and consistent with the above scaling relations between them. The distribution of $\Delta$, shown in Fig. 3 for different $s_{\max }$, has been fitted to $P(\Delta) \sim \Delta^{-\gamma}$ using a maximum-likelihood approach [49]. The estimated exponent $\hat{\gamma}\left(s_{\max }\right)$ changes from high values $\left[\gamma_{\mathrm{I}}=2.6(3)\right.$ for $s_{\max }=$ 2.3 ] to a lower value $\gamma_{\text {II }}=1.27(5)$ for $s_{\max } \lesssim 2.2$.

Rather than a true variation in $\gamma\left(s_{\max }\right)$, the behavior is a consequence of the superposition of two populations with 


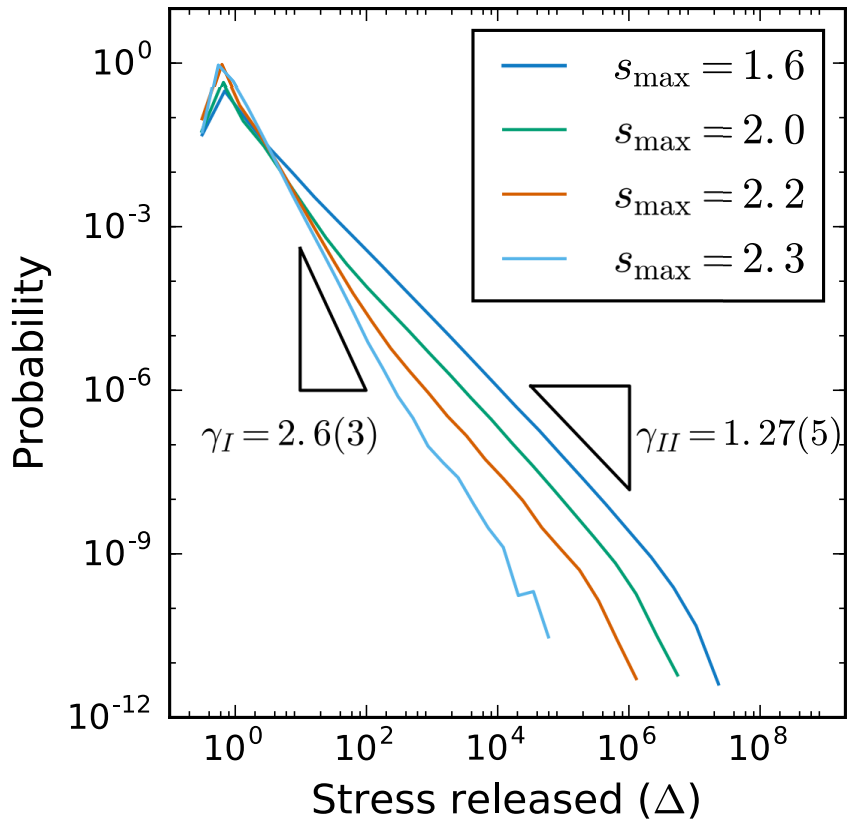

FIG. 3. Distributions of $\Delta$ for different $s_{\max }$ values. Each catalog contains $n=5 \times 10^{5}$ events.

$\operatorname{high}\left(\gamma_{\mathrm{I}}\right)$ and low $\left(\gamma_{\mathrm{II}}\right)$ exponents:

$$
P\left(\Delta \mid s_{\max }\right)=x_{\mathrm{I}}\left(s_{\max }\right) P_{I}\left(\Delta \mid s_{\max }\right)+x_{\mathrm{II}}\left(s_{\max }\right) P_{\mathrm{II}}\left(\Delta \mid s_{\max }\right),
$$

where $x_{\mathrm{II}}$ and $x_{\mathrm{I}}=1-x_{\mathrm{II}}$ are the fraction of events, dependent on $s_{\max }$. This superposition gives rise to an "effective" exponent $\gamma\left(s_{\max }\right)$. A separation between the two populations can be obtained by thresholding $p_{p}$ at the fracture initiation site. Figure 4(a) shows an example for $s_{\max }=2.0$ by separating the events initiated at sites $i$ with $p_{p, i}$ higher and lower than a pressure threshold $p_{\text {th }}=0.7$. Figure 4(b) maps the location of the two populations of fractures, simplified here in elliptical shapes accounting for their size, anisotropy, and orientation. Due to the point diffusion imposed by the pressure profiles, the separation can be reinterpreted in terms of the metric distance from the injection site. Population II is initiated inside a highly stressed area close to the injection site. Events I occur far from the injection site where both $p_{p}$ and stress levels are low; that is, $p_{\text {th }}$ increases with $s_{\max }$. Figure 4(c) displays the exponents of both populations for different values of $s_{\max }$. Events generated at $p_{p}>p_{\text {th }}$ can be classified as II with $\gamma_{\text {II }} \sim 1.27$, while events with $p_{p}<p_{\text {th }}$ make up population I with $\gamma_{\mathrm{I}} \sim 2.6$. The effective exponent of $P\left(\Delta \mid s_{\max }\right)$ undergoes a sharp transition between these regimes at $s_{\max }=2.2$. Lower event numbers as typically encountered in field data lead to a more gradual effective transition, as shown, for example, by the results for $N=10^{5}$ (green dashed curve).

To investigate the differences between the two populations further, Fig. 5 shows the evolution of the cumulative number of events and slips for both populations. While the total number of events, and their proportion $x_{\mathrm{II}}$, is proportional to the injected volume, only in the case of population $I$ is this proportionality preserved in the cumulative number of slips, denoting a stationary distribution of magnitudes. In contrast, population II exhibits a quadratic increase with injected volume. We attribute the difference in the scaling relations to the
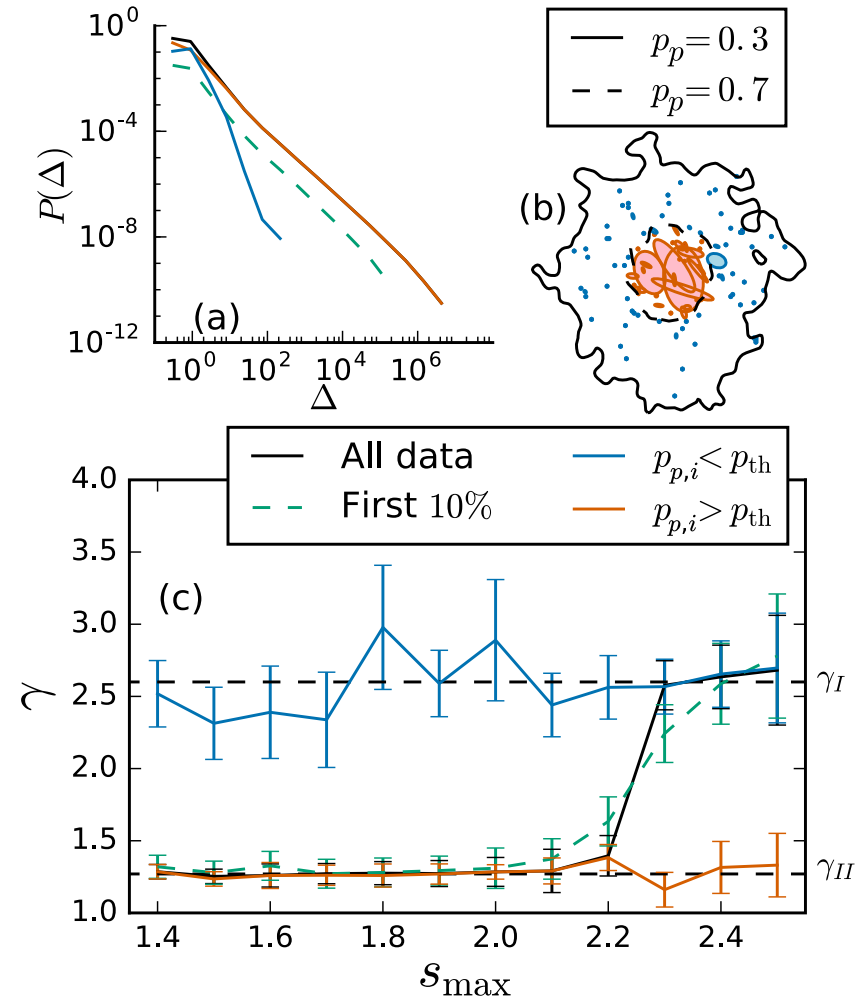

FIG. 4. (a) Slip distribution of fractures for $s_{\max }=2.0$ of all data (black), first $10 \%$ of events (green), and events initiating with high pressure $p_{p, i}$ above (red) and below (blue) $p_{\text {th }}=0.7$. (b) Spatial contour lines of the percolated area $\left(p_{p, r}=p_{\min }=0.3\right.$, solid curve) and for $p_{\mathrm{th}}=0.7$ (dashed curve) after $10^{4}$ injection steps. Fracture areas of the last 100 events are represented as ellipses and classified as I (blue) and II (red) from their corresponding $p_{p, i}$. (c) Estimated exponents $\hat{\gamma}$ for populations I (blue) and II (red) and total population (black solid curve) at different $s_{\max }$ for $N=10^{6}$ and the first $N=10^{5}$ events (green dashed curve). Values $\gamma_{\text {I }} \sim 2.6$ and $\gamma_{\text {II }} \sim 1.27$ are shown as black dashed lines.

fact that the finite extension of the stimulated reservoir affects the two populations differently. Since $\gamma_{\mathrm{I}}>2$, the mean stress released by population I does not significantly depend on the stimulated area. Conversely, since $\gamma_{\mathrm{II}}<2$, the opposite is true for population II. Indeed, $P_{\mathrm{II}}(\Delta)$ exhibits an exponential cutoff at a characteristic $\Delta_{c}$ (Fig. 3). Specifically, above a minimum value $\Delta_{m}$

$$
P_{\mathrm{II}}\left(\Delta ; \gamma_{\mathrm{II}}, \Delta_{c}, \Delta_{m}\right) d \Delta=\frac{\Delta^{-\gamma_{\mathrm{II}}} \exp \left(-\Delta / \Delta_{c}\right)}{\Delta_{c}^{1-\gamma_{\mathrm{II}}} \Gamma\left(1-\gamma_{\mathrm{II}}, \frac{\Delta_{m}}{\Delta_{c}}\right)} d \Delta,
$$

where $\Gamma$ is the upper incomplete gamma function. We estimate the cutoff values using maximum likelihood based on Eq. (8) over events larger than $\Delta_{m}=10^{3}$ by imposing $\gamma_{\mathrm{II}}=$ 1.27. Note that the range of $\Delta>\Delta_{m}=10^{3}$ excludes population I for all explored values of $s_{\max }$ and $N$. The estimation of the cutoff for $\Delta$ reveals a power-law dependence on the injection volume $V$ :

$$
\Delta_{c}=\lambda_{\Delta} V^{\eta_{\Delta}} .
$$

The estimated values of the scalar term $\lambda_{\Delta}$ and the exponent $\eta_{\Delta}$ using a regression approach $\left[\hat{\lambda}_{\Delta}\left(s_{\max }\right), \hat{\eta}_{\Delta}\left(s_{\max }\right)\right]$ are given 

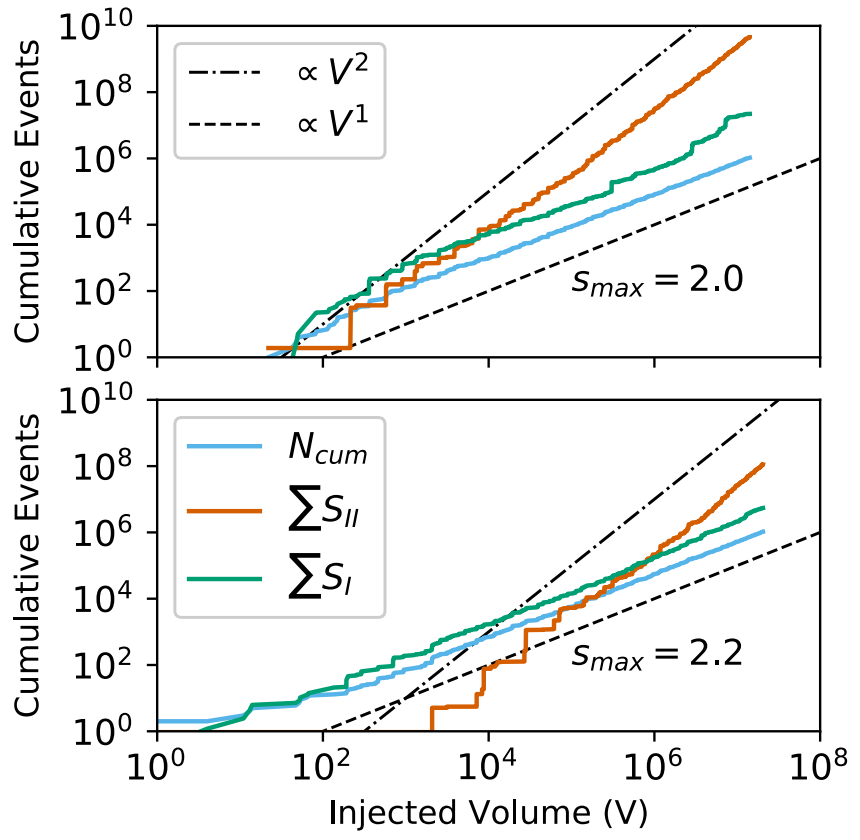

FIG. 5. Cumulative number of events and cumulative total slip for populations I and II, respectively, as a function of injected volume for a single simulation run with $s_{\max }=2.0$ (top) and $s_{\max }=2.2$ (bottom). Specifically, $N_{\text {cum }} \propto V$ and $\sum S_{I I} \propto V^{2}$ with horizontal offsets depending on $s_{\max }$. When separating events into populations I and II, events initiating within $p_{\text {th }} \pm 0.1 p_{\text {th }}$ were removed for visual clarity.

in Fig. 6. The estimated values $\hat{\lambda}_{\Delta}\left(s_{\max }\right)$ tend to decrease with $s_{\max }$, while $\hat{\eta}_{\Delta}\left(s_{\max }\right)$ is largely independent of $s_{\max }$ and fluctuates around $\eta_{\Delta} \sim 1.5$. As Fig. 6 shows, we obtain qualitatively similar results if the analysis is performed in terms of $A$ (with $A_{m}=10^{3}$ ), and we find $\eta_{A} \sim 0.9$. Note that the ratio $\eta_{A} \sim 0.64 \eta_{\Delta}$, which is consistent with the value $\xi \approx 2 / 3$ found for large values in Fig. 2.

Finally, Eq. (8) together with Eq. (9) and $\gamma_{\mathrm{II}}=1.27$ allows us to estimate $x_{\mathrm{II}}\left(s_{\max }\right)$ in Eq. (7) from the ratio $P(\Delta) / P_{\mathrm{II}}\left[\Delta ; \hat{\gamma}_{\mathrm{II}}, \hat{\lambda}_{\Delta}\left(s_{\max }\right) V^{\hat{\eta}_{\Delta}\left(s_{\max }\right)}, \Delta_{m}\right]$ within the interval $\Delta_{m}<\Delta<\Delta_{c}$. As Fig. 7 shows, the fraction $x_{\text {II }}$ decreases with $s_{\max }$ and appears to be stationary in time although the accelerated drop at $s_{\max }=2.2$, matching the transition in Fig. 3, might get sharper for longer times, i.e., larger stimulated areas. Notice that $x_{\mathrm{II}}\left(s_{\max }\right)$ largely coincides with the ratio $N_{i} / N$, where $N_{i}$ is the number of events satisfying $p_{i}>p_{\text {th }}$, used in Fig. 4.

While we presented results for fixed $s_{\min }$ and $p_{p \text {,min }}$, we found that the reported phenomenology is qualitatively robust over a wide range of $s_{\min }$ and $p_{p, \min }$, including arbitrarily close to $p_{p, \min }=0$. Only close to the percolation limit $\left(p_{p, \min }=\right.$ 1) does the behavior change fundamentally [40]. Including dissipation led to a decrease in $\Delta_{c}$ but did not affect the scaling. Together, these observations imply a certain degree of universality from the fluid regime, where viscous forces dominate, to intermediate regimes, where capillary forces become comparable to viscous forces. The value of $\gamma_{\mathrm{II}}=$ 1.27(5) is compatible with the critical flow regime of the same stick-slip geomechanical model [Eq. (6)] under shear stress driving (see Fig. 8), which is thought to represent tectonic

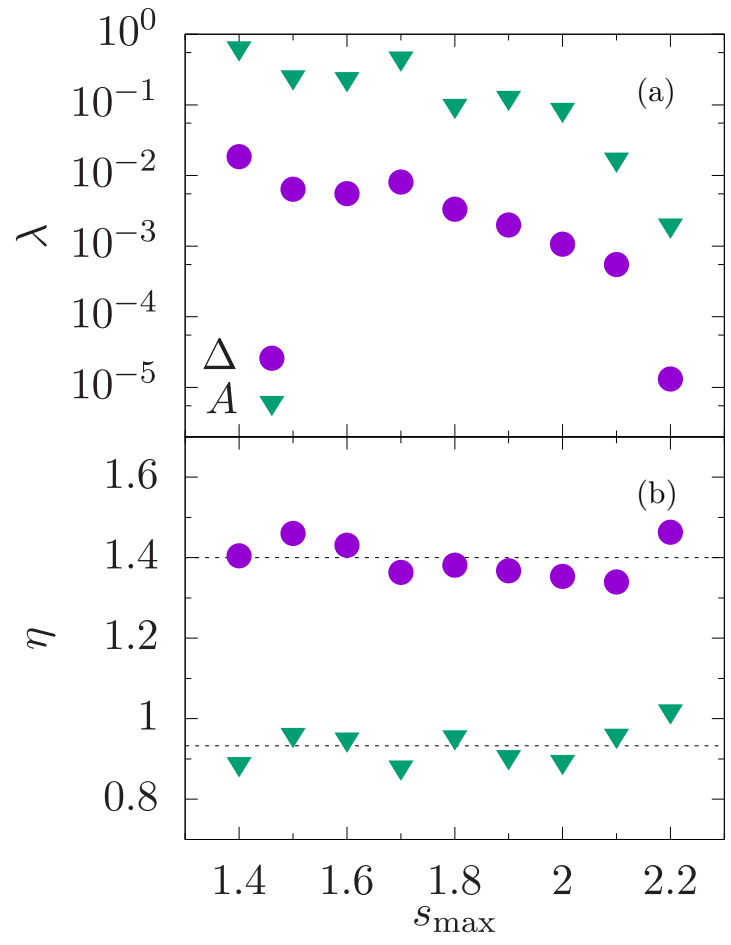

FIG. 6. Variations in the cutoff of population II as parametrized by Eq. (9): Estimated values of (a) $\lambda$ and (b) $\eta$ for stress release (purple dots) and areas (green triangles), respectively, i.e., $\Delta_{c}=\lambda_{\Delta} V^{\eta_{\Delta}}$ and $A_{c}=\lambda_{A} V^{\eta_{A}}$. The cutoff in the released stress by population II scales with exponent $\eta_{\Delta}=1.4(1)$, while the one for the area scales with exponent $\eta_{A}=0.9(1)$.

seismicity [44]. Yet, under such homogeneous driving the stick-slip model cannot reproduce events I in the steady-state regime. This strongly suggests that the evolving heterogeneities, here generated by the propagating front and pore-pressure profile, are necessary for the stationary coexistence of the two populations. Specifically, in our model, events II occur inside a region where the critical flow regime of the stick-slip model is reached due to high $p_{p}$. In contrast, population I consists of events generated at lower stress levels. Simulations at low $s_{\max }$ are from the beginning closer to criticality than higher $s_{\max }$ simulations that need larger injection volumes to stimulate population II. This also explains why population I is not likely to be observed in tectonic settings, where the initial loading is high. Moreover, numerical

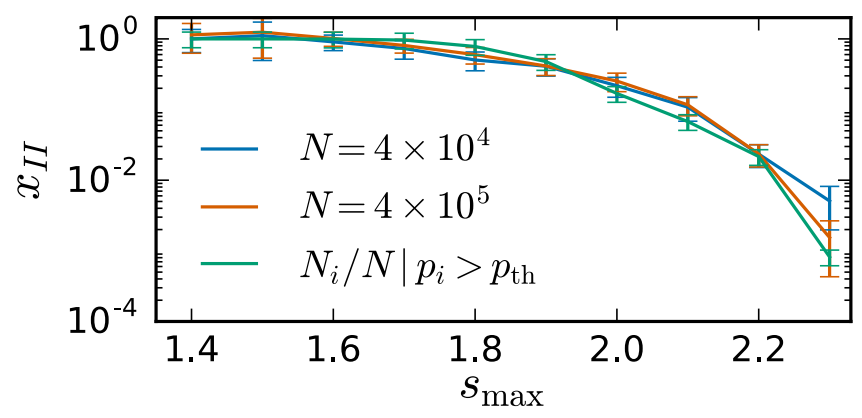

FIG. 7. Estimated fraction $x_{\text {II }}\left(s_{\max }\right)$ for different $N$ using Eqs. (7) and (8) and by thresholding $p_{p, i}>p_{\text {th }}$ for $N=4 \times 10^{5}$. 


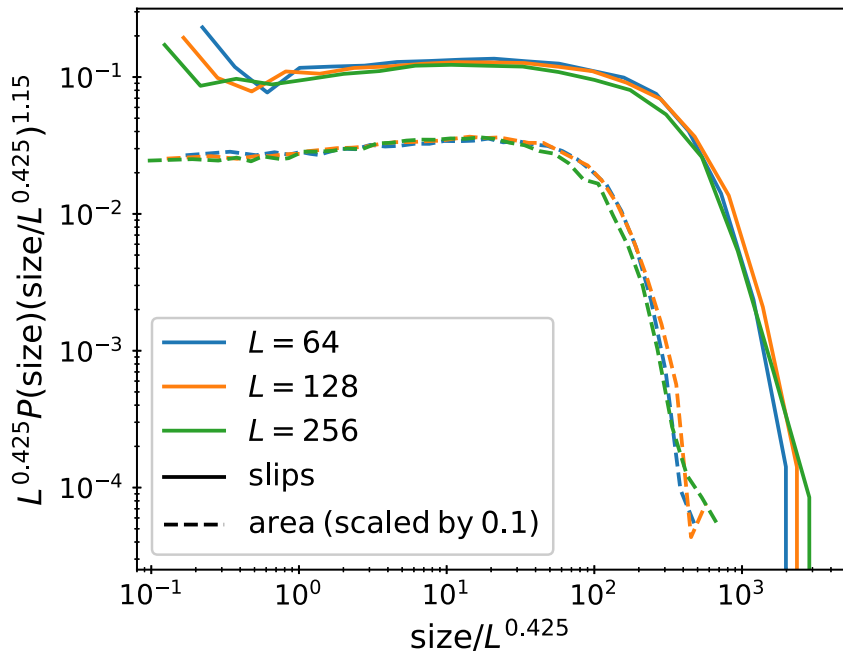

FIG. 8. Rescaled distribution of event sizes measured with fracture area $A$ and number of slips $S$ for different lattice sizes subject to uniform tectonic loading. Note that the rescaled distributions of both $A$ and $S$ have been shifted down for better readability. The exponent $\gamma \approx 1.15$ is compatible with $\gamma_{\text {II }}$ of population II in our fluid-driven model. Measured event sizes are still within the proportionality regime observed in Fig. 2(a). Note that locally, and under quasistatic driving, fracture propagation only depends on the internal state of the reservoir. Given the similarity in critical exponents, dry critical fractures initiated under homogeneous shear stress driving seem to be indistinguishable from wet critical population II fractures in our model.

simulations confirmed that if the medium is already critically preloaded through tectonic driving, then fluid stimulation will lead to events II but with a stationary size distribution with $\gamma_{\text {III }}$, where $\Delta_{c}$ is set by the overall system size. This is indistinguishable from events due to tectonic driving (Fig. 8) obtained from the same stick-slip model at $p_{p, i}=0$ with a constant shear stress driving $d \tau_{s, i} / d t$.

\section{DISCUSSION}

The conversion of critical exponents in geomechanical variables to $b$ values for moment magnitudes $m$ remains an open question. The Hanks-Kanamori relationship established for large earthquakes, $m=\frac{2}{3} \log _{10} M_{0}+C$ [50], provides a rough approximation though it is problematic for microseismicity [51,52]. It implies a moment-magnitude scaling $N \sim$ $M_{0}^{-\frac{2}{3} b}$. In our simulations, the number of events with stress released greater than $\Delta$ is $N \sim \Delta^{-(\gamma-1)}$. Assuming that the shear modulus $\mu$ is constant, $M_{0}=\mu P_{0} \sim \Delta$ [48], and equating the two expressions for $N$ yields $b=\frac{3}{2}(\gamma-1)$. This rough approximation gives $b_{\mathrm{II}}=0.41 \pm 0.08$ and $b_{\mathrm{I}}=2.4 \pm 0.5$. However, if we assume a correspondence between model simulations of tectonic driving and natural settings, the exponent match in simulations between population II and tectonic driving suggests that $b_{\text {II }}$ should be closer to 1 . Our results agree qualitatively with the observed range of $b$ and its dependence on stress or depth [53] but currently cannot provide an exact estimation of $b$ based on $\gamma$.

Despite this, based on the behavior of our model as captured by Eqs. (7)-(9), we can develop a general methodology

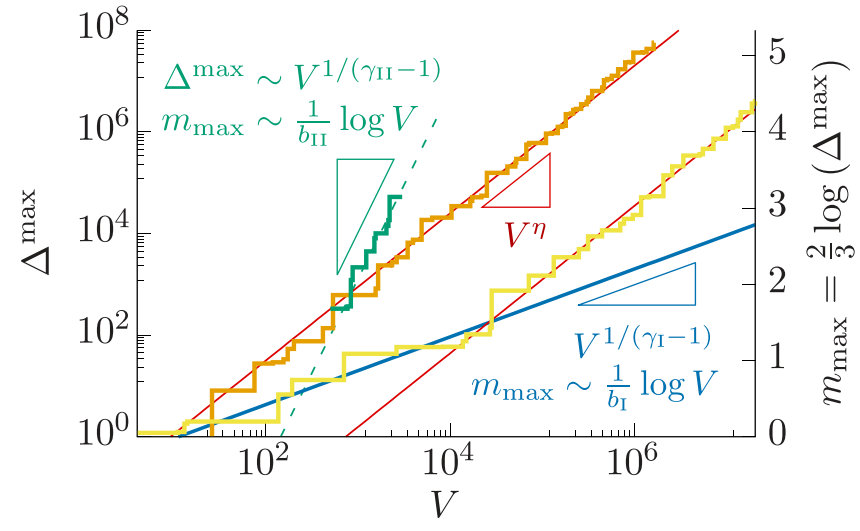

FIG. 9. Expected stress release of maximum event $\Delta^{\text {max }}$ (straight lines) using fitted values $\hat{\eta}, \hat{\lambda_{\Delta}}, \hat{\gamma_{I}}, \hat{\gamma_{I I}}$, and $\hat{x_{I I}}$ compared with records of direct numerical simulations (steps) as a function of injected volume $V$ (or time) for $s_{\max }=2.2$ (yellow), $s_{\max }=1.4$ (orange), and uniform tectonic loading (green).

to quantify the seismic hazard of strong events in different standard ways using theoretical and numerical forecasts. The increase in the expected maximum or record event size as a function of injected volume has been proposed as an effective measurement for traffic-light systems in industrial operations [8]. Figure 9 shows three different scenarios in our model, each of which can be mapped to one of the paradigms (paradigms A-C) presented in the introduction. Given a stationary cumulative size distribution $F(\Delta)$, records increase with the total number of events $N_{0}$ as $N_{0} P(\Delta)[F(\Delta)]^{N_{0}-1}$. For a pure power-law distribution with exponent $\gamma$ and considering $N_{0} \propto V$ (e.g., Figs. 5 and 10), which applies to A-type and C-type hazard models, this gives

$$
\Delta^{\max } \sim V^{\frac{1}{\gamma-1}} .
$$

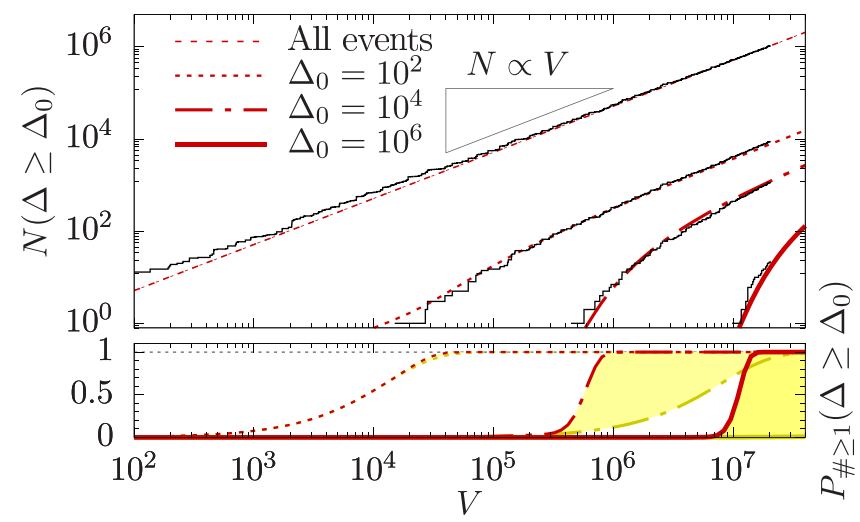

FIG. 10. Top: Expected (red) and directly observed (black) cumulative number of events above different thresholds $\Delta_{0}$ for $s_{\max }=$ 2.2 as a function of injected volume $V$. Note that higher thresholds are reached at later times. Bottom: Probability of observing the first event above $\Delta_{0}$ [i.e., $N\left(\Delta \geqslant \Delta_{0}\right)=1$ in top panel] before injecting $V$ estimated based on our model (red curves) and a forecast with constant $d N_{\Delta_{0}} / d V$ extrapolated from population I (brown curves) for comparison, both for $s_{\max }=2.2$. Colored areas highlight the difference between both estimates. 
This behavior is found in situations dominated by population I, with exponent $\gamma_{\mathrm{I}}$ (blue line in Fig. 9), and in critically loaded settings with $\gamma_{\text {II }}$ (green dashed line). In the former case, physical constrains that could potentially lead to cutoffs are not observed because extremely large events are rare due to the large exponent, $\gamma_{\mathrm{I}}>2$. Therefore A-type hazard models are adequate to capture situations in our model of fluid-induced seismicity when population I dominates. In contrast, when population II dominates, corresponding to low $s_{\max }$ or long injection times, large events are common and constrained by $\Delta_{c}$, which increases with $V$ according to Eq. (9) with $\eta \sim 3 / 2$ (red line) corresponding to paradigm B and consistent with Ref. [41]. Stationary distributions fulfilling Eq. (10) with exponent values $\gamma_{\mathrm{II}}<2$ are only observable if the magnitude is unconstrained. This happens in critically prestressed regions in our model of fluid-induced seismicity representing tectonic settings, as considered in paradigm $\mathrm{C}$.

The seismic hazard can also be quantified by the (expected) number of events $\left(N_{>\Delta_{0}}\right)$ with $\Delta>\Delta_{0}$, as a function of injected volume (see Fig. 10). Inspired by the proportionality between $N_{\Delta_{0}}$ and $V$ found in models within paradigm $\mathrm{A}$ and early field studies, Ref. [32] developed the concept of the seismogenic index $\Sigma$ in detail. Following (2), the seismogenic index is a single parameter able to characterize the seismic risk above any magnitude threshold $m_{0}$. Considering GR statistics,

$$
\Sigma:=\log _{10} N_{>m_{0}}-\log _{10} V+b\left(m_{0}-m_{c}\right) .
$$

In our units, we find that the condition $N_{>\Delta_{0}} \propto V$ is valid for any $\Delta_{0}$ as long as $V$ is sufficiently large, ensuring $\Delta_{c}(V)>$ $\Delta_{0}$ [Eq. (9)], but not at shorter stimulation times where $\Delta_{c}(V) \approx \Delta_{0}$. This is a consequence of the nonstationary size distribution of population II. At any injection volume, some size range exhibits a nonstatic $d N_{\Delta_{0}} / d V$, e.g., for $\Delta_{0}=10^{4}$ in Fig. 10, consistent with paradigm B. This is not the case in critically loaded settings (paradigm C) and for relatively low stress levels and low injection volumes, also known as population I (paradigm A), both of which have stationary size distributions and $d N_{\Delta_{0}} / d V \equiv$ const (Fig. 5). Indeed, paradigm $\mathrm{B}$ is, in part, inspired by an underestimation of the true seismic hazard when $d N_{\Delta_{0}} / d V$ is extrapolated from the seismogenic indexes and distributions at low injection volumes. In particular, this observation led to the introduction of dependencies of the magnitude distribution on the injected volume, hypothesized to be caused by the finite size of the stimulated area [34,35]. In our model, this dependence is observed as well in low-stress regions after persistent fluid injection for large $\Delta_{0}$, as highlighted in the bottom panel of Fig. 10. In that case, population II localizes close to the injection source, supporting the ideas presented in Ref. [34].
The transition in the statistics of large events from population I to population II could explain as well the temporal decrease in $b$ reported in fluid-induced episodes of seismicity [54,55]. If the applicability of our model can be directly establishedpotentially with the help of laboratory experiments and/or measurements of the local stress field and the heterogeneity of the medium - the existence of and competition between these two populations provides a direct improvement of current hazard assessment techniques. Specifically, one could take advantage of the maximum-likelihood estimation of the parameters defining (7)-(9) or adapted techniques.

\section{CONCLUSIONS}

To summarize, our conceptual model can reproduce the main observed features of fluid-induced seismicity with respect to the GR distribution and the associated seismic hazard. In particular, it suggests that the observed range of $b$ valueswhere Eq. (1) is typically seen as a trademark of criticality [56-58], since the magnitude is a logarithmic measure of the released seismic energy [59]-arise from a crossover phenomenon, and it provides a clear interpretation of the different proposed paradigms in both micromechanical and statistical hazard modeling. The model offers a dynamical explanation of the variations in $b$ rooted in the underlying interactions involving fractures and fluid dynamics, in contrast to other explanations such as mechanical layering $[27,60]$, fractal elastic properties [61], or finite size effects [35]. Moreover, both field $[22,24]$ and laboratory [62-64] observations suggest that the diversity and variability in $b$ values might be related to the interplay between the stress levels and the heterogeneity in $s_{i}$, though this feature has not been reproduced by any valid model study up to now. Finally, the existence of populations with different GR characteristics is reminiscent of seismicity associated with volcanic activity, where a temporal switching between $b$ values has been observed and fluids are likely to play an important role as well [65]. Investigating this connection further remains an interesting challenge for the future.

Field data were not created for this research. All mentioned field data supporting the conclusions can be obtained from the references. All details of the conceptual model-including the relevant algorithms and how the model simulations were performed - can be obtained from this paper.

\section{ACKNOWLEDGMENTS}

This work was supported by funding from a Collaborative Research and Development grant from the Natural Sciences and Engineering Research Council of Canada. C.L.-M. was supported by the University of Calgary's PURE program. J.B. is thankful for financial support from AXA Research Fund for the project RheMechFail.
[1] D. W. Eaton, Passive Seismic Monitoring of Induced Seismicity (Cambridge University Press, Cambridge, 2018).

[2] S. A. Shapiro, Fluid-Induced Seismicity (Cambridge University Press, Cambridge, 2015), p. 276.
[3] T. H. Goebel and E. E. Brodsky, The spatial footprint of injection wells in a global compilation of induced earthquake sequences, Science 361, 899 (2018).

[4] T. A. Tafti, M. Sahimi, F. Aminzadeh, and C. G. Sammis, Use of microseismicity for determining the structure of the fracture 
network of large-scale porous media, Phys. Rev. E 87, 032152 (2013).

[5] A. Zang, V. Oye, P. Jousset, N. Deichmann, R. Gritto, A. McGarr, E. Majer, and D. Bruhn, Analysis of induced seismicity in geothermal reservoirs: An overview, Geothermics 52, 6 (2014).

[6] M. Schoenball, N. C. Davatzes, and J. M. Glen, Differentiating induced and natural seismicity using space-time-magnitude statistics applied to the Coso Geothermal field, Geophys. Res. Lett. 42, 6221 (2015).

[7] P. Martínez-Garzón, G. Kwiatek, M. Bohnhoff, and G. Dresen, Volumetric components in the earthquake source related to fluid injection and stress state, Geophys. Res. Lett. 44, 800 (2017).

[8] G. Kwiatek, T. Saarno, T. Ader, F. Bluemle, M. Bohnhoff, M. Chendorain, G. Dresen, P. Heikkinen, I. Kukkonen, P. Leary, M. Leonhardt, P. Malin, P. Martínez-Garzón, K. Passmore, P. Passmore, S. Valenzuela, and C. Wollin, Controlling fluidinduced seismicity during a 6.1-km-deep geothermal stimulation in Finland, Sci. Adv. 5, eaav7224 (2019).

[9] S. Shapiro and C. Dinske, Scaling of seismicity induced by nonlinear fluid-rock interaction, J. Geophys. Res.: Solid Earth 114, B09307 (2009).

[10] S. Maghsoudi, J. Baró, A. Kent, D. Eaton, and J. Davidsen, Interevent triggering in microseismicity induced by hydraulic fracturing, Bull. Seismol. Soc. Am. 108, 1133 (2018).

[11] R. Schultz, G. Atkinson, D. W. Eaton, Y. J. Gu, and H. Kao, Hydraulic fracturing volume is associated with induced earthquake productivity in the Duvernay play, Science 359, 304 (2018).

[12] W. L. Ellsworth, Injection-induced earthquakes, Science 341, 1225942 (2013).

[13] N. B. Nagel, X. Garcia, M. A. Sanchez, and B. Lee, Understanding "SRV": A numerical investigation of "wet" vs. "dry" microseismicity during hydraulic fracturing, in SPE Annual Technical Conference and Exhibition, 8-10 October, San Antonio, Texas, USA (Society of Petroleum Engineers, Richardson, TX, 2012), Document SPE-159791-MS.

[14] N. Nagel, M. Sanchez-Nagel, F. Zhang, X. Garcia, and B. Lee, Coupled numerical evaluations of the geomechanical interactions between a hydraulic fracture stimulation and a natural fracture system in shale formations, Rock Mech. Rock Eng. 46, 581 (2013).

[15] S. Maxwell, M. Mack, F. Zhang, D. Chorney, S. Goodfellow, and M. Grob, Differentiating wet and dry microseismic events induced during hydraulic fracturing, in Unconventional Resources Technology Conference, San Antonio, Texas, 20-22 July 2015 (Society of Petroleum Engineers, Richardson, TX, 2015), pp. 1513-1524.

[16] S. Maxwell, D. Chorney, and S. Goodfellow, Microseismic geomechanics of hydraulic-fracture networks: Insights into mechanisms of microseismic sources, Leading Edge 34, 904 (2015).

[17] N. R. Warpinski, J. Du, and U. Zimmer, Measurements of hydraulic-fracture-induced seismicity in gas shales, SPE Prod. Oper. 27, 240 (2012).

[18] S. Maxwell, M. Jones, R. Parker, S. Miong, S. Leaney, D. Dorval, D. D’Amico, J. Logel, E. Anderson, and K. Hammermaster, Fault activation during hydraulic fracturing, in SEG Technical Program Expanded Abstracts 2009 (Society of Exploration Geophysicists, Tulsa, 2009), pp. 1552-1556.
[19] X. Bao and D. W. Eaton, Fault activation by hydraulic fracturing in western Canada, Science 354, 1406 (2016).

[20] K.-H. Kim, J.-H. Ree, Y. Kim, S. Kim, S. Y. Kang, and W. Seo, Assessing whether the $2017 M_{\mathrm{W}} 5.4$ Pohang earthquake in South Korea was an induced event, Science 360, 1007 (2018).

[21] S. Wiemer and M. Wyss, Mapping spatial variability of the frequency-magnitude distribution of earthquakes, in $\mathrm{Ad}$ vances in Geophysics (Elsevier, Burlington, 2002), Vol. 45, pp. 259-302.

[22] D. Schorlemmer, S. Wiemer, and M. Wyss, Variations in earthquake-size distribution across different stress regimes, Nature (London) 437, 539 (2005).

[23] C. Gu, A. Y. Schumann, M. Baiesi, and J. Davidsen, Triggering cascades and statistical properties of aftershocks, J. Geophys. Res.: Solid Earth 118, 4278 (2013).

[24] C. H. Scholz, On the stress dependence of the earthquake $b$ value, Geophys. Res. Lett. 42, 1399 (2015).

[25] J. Davidsen and M. Baiesi, Self-similar aftershock rates, Phys. Rev. E 94, 022314 (2016)

[26] C. Dinske and S. A. Shapiro, Seismotectonic state of reservoirs inferred from magnitude distributions of fluid-induced seismicity, J. Seismol. 17, 13 (2013).

[27] D. W. Eaton, J. Davidsen, P. K. Pedersen, and N. Boroumand, Breakdown of the Gutenberg-Richter relation for microearthquakes induced by hydraulic fracturing: Influence of stratabound fractures, Geophys. Prospect. 62, 806 (2014).

[28] D. W. Eaton and N. Igonin, What controls the maximum magnitude of injection-induced earthquakes? Leading Edge 37, 135 (2018).

[29] R. Schultz, V. Stern, and Y. J. Gu, An investigation of seismicity clustered near the Cordel Field, west central Alberta, and its relation to a nearby disposal well, J. Geophys. Res.: Solid Earth 119, 3410 (2014).

[30] A. McGarr, Seismic moments and volume changes, J. Geophys. Res. 81, 1487 (1976).

[31] A. McGarr, Maximum magnitude earthquakes induced by fluid injection, J. Geophys. Res.: Solid Earth 119, 1008 (2014).

[32] S. A. Shapiro, C. Dinske, C. Langenbruch, and F. Wenzel, Seismogenic index and magnitude probability of earthquakes induced during reservoir fluid stimulations, Leading Edge 29, 304 (2010).

[33] C. Frohlich, Two-year survey comparing earthquake activity and injection-well locations in the Barnett Shale, Texas, Proc. Natl. Acad. Sci. USA 109, 13934 (2012).

[34] S. A. Shapiro, O. S. Krüger, C. Dinske, and C. Langenbruch, Magnitudes of induced earthquakes and geometric scales of fluid-stimulated rock volumes, Geophysics 76, WC55 (2011).

[35] S. A. Shapiro, O. S. Krüger, and C. Dinske, Probability of inducing given-magnitude earthquakes by perturbing finite volumes of rocks, J. Geophys. Res.: Solid Earth 118, 3557 (2013).

[36] T. Dahm, S. Cesca, S. Hainzl, T. Braun, and F. Krüger, Discrimination between induced, triggered, and natural earthquakes close to hydrocarbon reservoirs: A probabilistic approach based on the modeling of depletion-induced stress changes and seismological source parameters, J. Geophys. Res.: Solid Earth 120, 2491 (2015).

[37] N. J. van der Elst, M. T. Page, D. A. Weiser, T. H. Goebel, and S. M. Hosseini, Induced earthquake magnitudes are as large as (statistically) expected, J. Geophys. Res.: Solid Earth 121, 4575 (2016). 
[38] K. Mogi, On the pressure dependence of strength of rocks and the Coulomb fracture criterion, Tectonophysics 21, 273 (1974).

[39] S. A. Miller, Y. Ben-Zion, and J.-P. Burg, A three-dimensional fluid-controlled earthquake model: Behavior and implications, J. Geophys. Res.: Solid Earth 104, 10621 (1999).

[40] J. Q. Norris, D. L. Turcotte, and J. B. Rundle, Loopless nontrapping invasion-percolation model for fracking, Phys. Rev. E 89, 022119 (2014).

[41] M. Galis, J. P. Ampuero, P. M. Mai, and F. Cappa, Induced seismicity provides insight into why earthquake ruptures stop, Sci. Adv. 3, eaap7528 (2017).

[42] S. Whitaker, Flow in porous media I: A theoretical derivation of Darcy's law, Transp. Porous Media 1, 3 (1986).

[43] D. Wilkinson and J. F. Willemsen, Invasion percolation: A new form of percolation theory, J. Phys. A: Math. Gen. 16, 3365 (1983).

[44] Y. Ben-Zion and J. R. Rice, Earthquake failure sequences along a cellular fault zone in a three-dimensional elastic solid containing asperity and nonasperity regions, J. Geophys. Res.: Solid Earth 98, 14109 (1993).

[45] The trapping rule is introduced as a reasonable physical constraint in the invasion percolation in 2D against an incompressible fluid. The 2D representation in our model is a simplification to mimic the "almost" planar propagation of fluids and the corresponding alignment with the direction of maximum shear stress observed in field experiences. In the real setting the reservoir itself is 3D. This "almost" in the definition of planar is enough to provide escape routes for the "trapped" reservoir fluids. For this reason, the trapping rule, which only makes sense in a pure 2D system, was regarded as inadequate for our model.

[46] Y. Ben-Zion, On quantification of the earthquake source, Seismol. Res. Lett. 72, 151 (2001).

[47] D. S. Fisher, K. Dahmen, S. Ramanathan, and Y. Ben-Zion, Statistics of Earthquakes in Simple Models of Heterogeneous Faults, Phys. Rev. Lett. 78, 4885 (1997).

[48] Y. Ben-Zion and L. Zhu, Potency-magnitude scaling relations for southern California earthquakes with $1.0<M_{L}<7.0$, Geophys. J. Int. 148, F1 (2002).

[49] J. Baró and E. Vives, Analysis of power-law exponents by maximum-likelihood maps, Phys. Rev. E 85, 066121 (2012).

[50] T. C. Hanks and K. Hiroo, A moment magnitude scale, J. Geophys. Res.: Solid Earth 84, 2348 (1979).

[51] T. C. Hanks and D. M. Boore, Moment-magnitude relations in theory and practice, J. Geophys. Res.: Solid Earth 89, 6229 (1984).
[52] M. Zecevic, S. Grant, D. W. Eaton, and J. Davidsen, How do seismic event sizes scale in the microseismic range? in GeoConvention 2017, Calgary, Canada, 15-19 May 2017 (Canadian Society of Petroleum Geologists, Calgary, 2017), Poster 057_GC2017.

[53] M. Kozłowska, M. R. Brudzinski, P. Friberg, R. J. Skoumal, N. D. Baxter, and B. S. Currie, Maturity of nearby faults influences seismic hazard from hydraulic fracturing, Proc. Natl. Acad. Sci. USA 115, E1720 (2018).

[54] C. E. Bachmann, S. Wiemer, B. P. Goertz-Allmann, and J. Woessner, Influence of pore-pressure on the event-size distribution of induced earthquakes, Geophys. Res. Lett. 39, L09302 (2012).

[55] S. Cesca, F. Grigoli, S. Heimann, A. González, E. Buforn, S. Maghsoudi, E. Blanch, and T. Dahm, The 2013 SeptemberOctober seismic sequence offshore Spain: A case of seismicity triggered by gas injection? Geophys. J. Int. 198, 941 (2014).

[56] P. Bak and K. Chen, Self-organized criticality, Sci. Am. 264, 46 (1991).

[57] D. L. Turcotte, Self-organized criticality, Rep. Prog. Phys. 62 1377 (1999).

[58] J. P. Sethna, K. A. Dahmen, and C. R. Myers, Crackling noise, Nature (London) 410, 242 (2001).

[59] D. L. Turcotte, Fractals and Chaos in Geology and Geophysics, 2nd ed. (Cambridge University Press, Cambridge, 1997).

[60] S. Maghsoudi, D. W. Eaton, and J. Davidsen, Nontrivial clustering of microseismicity induced by hydraulic fracturing, Geophys. Res. Lett. 43, 10672 (2016).

[61] C. Langenbruch and S. Shapiro, Gutenberg-Richter relation originates from Coulomb stress fluctuations caused by elastic rock heterogeneity, J. Geophys. Res.: Solid Earth 119, 1220 (2014).

[62] D. Amitrano, Brittle-ductile transition and associated seismicity: Experimental and numerical studies and relationship with the $b$ value, J. Geophys. Res.: Solid Earth 108, 2044 (2003).

[63] T. H. W. Goebel, D. Schorlemmer, T. W. Becker, G. Dresen, and C. G. Sammis, Acoustic emissions document stress changes over many seismic cycles in stick-slip experiments, Geophys. Res. Lett. 40, 2049 (2013).

[64] G. Kwiatek, T. H. W. Goebel, and G. Dresen, Seismic moment tensor and $b$ value variations over successive seismic cycles in laboratory stick-slip experiments, Geophys. Res. Lett. 41, 5838 (2014).

[65] N. S. Roberts, A. F. Bell, and I. G. Main, Mode switching in volcanic seismicity: El Hierro 2011-2013, Geophys. Res. Lett. 43, 4288 (2016). 